\title{
Multi-party Computation with Hybrid Security
}

\author{
Matthias Fitzi $^{1}$, Thomas Holenstein ${ }^{2}$, and Jürg Wullschleger ${ }^{3}$ \\ 1 Department of Computer Science \\ University of California, Davis \\ fitzi@cs.ucdavis.edu \\ 2 Department of Computer Science \\ ETH Zurich, Switzerland \\ holenst@inf .ethz.ch \\ 3 Département d'Informatique et Recherche Opŕationnelle \\ Université de Montréal, Canada \\ wullschj@iro.umontreal.ca
}

\begin{abstract}
It is well-known that $n$ players connected only by pairwise secure channels can achieve multi-party computation secure against an active adversary if and only if

$-t<n / 2$ of the players are corrupted with respect to computational security, or

$-t<n / 3$ of the players are corrupted with respect to unconditional security.

In this paper we examine to what extent it is possible to achieve conditional (such as computational) security based on a given intractability assumption with respect to some number $T$ of corrupted players while simultaneously achieving unconditional security with respect to a smaller threshold $t \leq T$. In such a model, given that the intractability assumption cannot be broken by the adversary, the protocol is secure against $T$ corrupted players. But even if it is able to break it, the adversary is still required to corrupt more than $t$ players in order to make the protocol fail.

For an even more general model involving three different thresholds $t_{p}$, $t_{\sigma}$, and $T$, we give tight bounds for the achievability of multi-party computation. As one particular implication of this general result, we show that multi-party computation computationally secure against $T<n / 2$ actively corrupted players (which is optimal) can additionally guarantee unconditional security against $t \leq n / 4$ actively corrupted players "for free."
\end{abstract}

Keywords: Broadcast, computational security, multi-party computation, unconditional security.

\section{Introduction}

Secure distributed cooperation among mutually distrusting players can be achieved by means of general multi-party computation (MPC). Typically, the goal of such a cooperation consists of jointly computing a function on the players' inputs in a way that guarantees correctness of the computation result while keeping 
the players' inputs private - even if some of the players are corrupted by an adversary.

Different models for MPC have been proposed in the literature with respect to communication, corruption flavor, and adversarial power. In this paper, we restrict our view to the following parameters.

COMmUnicATiOn: We exclusively consider synchronous networks meaning that, informally speaking, the players are synchronized to common communication rounds with the guarantee that a sent message will be delivered still during the same communication round.

CORRuption: We assume a central threshold adversary with respect to a given, fixed threshold $t$, meaning that it can select up to arbitrary $t$ out of the $n$ players and corrupt them (meaning to take control over them). Such a player is then said to be corrupted whereas a non-corrupted player is called correct. An active adversary (active corruption) corrupts players by making them deviate from the protocol in an arbitrarily malicious way.

SECURITY: A protocol achieves unconditional security if even a computationally unbounded adversary cannot make the protocol fail - except for some negligible error probability. A protocol achieves computational security if an adversary restricted to probabilistic polynomial time computations cannot make the protocol fail except for some negligible error probability. In this paper, we consider both kinds of security.

\subsection{Previous Work}

The MPC problem was first stated by Yao Yao82. Goldreich, Micali, and Wigderson [GMW87] gave the first complete solution to the problem with respect to computational security. For the model with a passive adversary (passive model, for short), and given pairwise communication channels, they gave an efficient protocol that tolerates any number of corrupted players, $t<n$. For the model with an active adversary (active model), and given both pairwise and broadcast channels, they gave an efficient protocol that tolerates any faulty minority, $t<n / 2$, which is optimal in the sense that no protocol exists for $t \geq n / 2$. Note that when not demanding security to the full extent, computationally secure MPC is also achievable in presence of an active adversary that corrupts $t \geq n / 2$ players GMW87, GHY87, BG89, GL90, FGH ${ }^{+} 02$, GL02, FHHW03. However, in this case, robustness cannot be guaranteed, i.e., it can not be guaranteed that every player receives a result [Cle86.

With respect to unconditional security, Ben-Or, Goldwasser, and Wigderson [BGW88], and independently, Chaum, Crépeau, and Damgård CCD88] gave efficient protocols for the passive model that tolerate $t<n / 2$ and protocols for the active model that tolerate $t<n / 3$ - assuming only pairwise communication channels in both cases. Both bounds are tight. Beaver Bea89, Rabin, and Ben-Or RB89] considered the active model when given both pairwise and broadcast channels among the players. They gave efficient protocols that achieve unconditional security for $t<n / 2$ which is optimal. A more efficient protocol for this model was given by Cramer et al. $\left.\mathrm{CDD}^{+} 99\right]$. 
With lack of better knowledge, protocols with computational security must be based on unproven intractability assumptions, i.e., they must build up on cryptographic primitives such as trapdoor permutations that are not known to exist. Furthermore, even if such primitives existed, the particular choice of a candidate implementation of such a primitive might be a bad one.

In order to prevent complete failure in these cases, Chaum Cha89] considered a "hybrid" security model for MPC that achieves computational security for some large threshold $T$ but, at the same time, unconditional security for some smaller threshold $t \leq T$ - meaning that, in order to make the protocol fail, the adversary must either corrupt more than $T$ players, or corrupt more than $t$ players but additionally be able to break the underlying computational hardness assumption. In the passive model, given pairwise communication channels, Chaum's protocol achieves computational security with respect to $T<n$ and unconditional security with respect to $t<n / 2$. Thus, this protocol simultaneously achieves the optimal bounds for computational and unconditional security.

In the active model, given pairwise and broadcast channels, his protocol achieves computational security with respect to $T<n / 2$ and additionally provides unconditional privacy for all players' inputs as long as up to $t<n / 3$ players are corrupted. Note that the later results in [Bea89, RB89, CDD ${ }^{+}$99] strictly imply this result: unconditional security for $t<n / 2$ when assuming broadcast 4 In WP89, the same "hybrid" model was considered with respect to the simulation of broadcast when given only pairwise communication channels.

\subsection{Multi-party Computation beyond $\mathrm{t}<\mathrm{n} / 3$ without Broadcast}

The active model for MPC tolerating at least $n / 3$ corrupted players typically assumes broadcast channels [GMW87, Bea89, RB89]. This is a very strong assumption and might not always be appropriate. Rather, broadcast has to be simulated by the players using the bilateral channels. But, without further assumptions, this simulation is only possible if $t<n / 3$ [LSP82, $\mathrm{DFF}^{+} 82$.

The only known way to allow for the simulation of broadcast beyond $t<n / 3$ is to use digital signatures [LSP82. However, it is important to note that digital signatures by themselves are not enough. It must be additionally guaranteed that all correct players verify each player's signatures in the same way, i.e., that all players hold the same list of public keys. Otherwise, the transfer of a signature would not be conclusive. We call such a setup a consistent publickey infrastructure (PKI). Such a PKI allows to efficiently simulate broadcast among the players secure against any number of corrupted players, $t<n$ [DS82]. Not only can a PKI be based on a computationally secure digital signature scheme but also on unconditionally secure pseudo-signatures PW96 and thus allowing for the simulation of unconditionally secure broadcast. Thus the results

\footnotetext{
${ }^{4}$ Note that Chaum's protocol still completely relies on cryptography since the protocol's correctness is only protected by cryptographic means, i.e., by breaking the cryptographic assumption the adversary can make the protocol fail by only corrupting one single player.
} 
in GMW87, Bea89, RB89, are equally achievable without broadcast channels but with an appropriate PKI to be set up among the players - computationally secure for GMW87] and unconditionally secure for the other cases.

We believe that assuming a PKI is more realistic than assuming broadcast channels among the players and thus follow this model.

It should be noted, though, that the use of unconditional pseudo-signatures is not very practical. The cost of broadcasting a single bit based on (computational) digital signatures is $t+1$ communication rounds and $O\left(n^{3} s\right)$ bits to be sent by all players during the protocol overall — where $s$ is the size of a signature [DS83]. The cost of broadcasting a bit using unconditional pseudo-signatures is $\Omega\left(n^{3}\right)$ rounds and $\Omega\left(n^{17}\right)$ bits to be sent overall [PW96 — which is still polynomial but nevertheless quite impractical 5

\subsection{Contributions}

Typical ways of setting up a PKI are to run a setup protocol among the players or to involve trust management over the Internet such as, e.g., the one in PGP. Evidently, both methods can fail to achieve a consistent PKI, namely, when to many players are corrupted, or, respectively, when the trust management is built on wrong assumptions. Thus, analogously to relying on computational security, relying on the consistency of a previously set-up PKI also imposes a potential security threat since the adversary might have been able to make the PKI inconsistent.

This raises the natural question of whether MPC relying on the consistency of a PKI and/or the security of a particular signature scheme can additionally guarantee unconditional security for the case where only a small number of the players are corrupted. Thus, in this paper, we extend the considerations in [Cha89] (regarding the active model) to the case where not only the adversary might be able to break the underlying hardness assumption but where also the PKI might be inconsistent.

In particular, we consider the following model for hybrid $M P C$ involving three thresholds $t_{p}, t_{\sigma}$, and $T$, where $t_{p}, t_{\sigma} \leq T$ with the following properties (see also Figure (1).

- If at most $f \leq \min \left(t_{p}, t_{\sigma}\right)$ players are corrupted then we demand unconditional security.

- If $f>t_{p}$ then we assume that the PKI is consistent, i.e., for $t_{p}<f \leq T$ the computation is only as secure as the PKI.

- If $f>t_{\sigma}$ then we assume that the adversary cannot forge signatures (except for some non-negligible probability), i.e., for $t_{\sigma}<f \leq T$ the computation is only as secure as the underlying signature scheme.

\footnotetext{
${ }^{5}$ Note that there is also a $(t+1)$-round variant with an overall bit complexity of approximately $\Theta\left(n^{6}\right)$. However, this variant is only a one-time signature scheme which basically means that the PKI only allows for a very limited number of signatures to be issued.
} 


\begin{tabular}{|c||l|}
\hline$f$ players corrupted & Security \\
\hline \hline$f \leq \min \left(t_{p}, t_{\sigma}\right)$ & unconditional \\
\hline$f \leq t_{\sigma} \wedge t_{p}<f \leq T$ & as secure as PKI, independent of signature scheme \\
\hline$f \leq t_{p} \wedge t_{\sigma}<f \leq T$ & as secure as signature scheme, independent of PKI \\
\hline$t_{p}, t_{\sigma}<f \leq T$ & as secure as PKI and signature scheme together \\
\hline
\end{tabular}

Fig. 1. Threshold conditions for hybrid MPC.

Or, in other words, if $f \leq t_{p}$ then the protocol must be secure even if the PKI is inconsistent, and, if $f \leq t_{\sigma}$ then the protocol must be secure even if the adversary is able to forge signatures. Thus, in order to make such a hybrid protocol fail with non-negligible probability, the adversary would have to corrupt more than $f=\min \left(t_{p}, t_{\sigma}\right)$ players and; having made for a bad PKI if $f>t_{p}$, or be able to forge signatures if $f>t_{\sigma}$.

Result. We show that hybrid MPC is achievable if and only if

$$
\left(2 T+t_{p}<n\right) \wedge\left(T+2 t_{\sigma}<n\right)
$$

implying that, without loss of generality, we can always assume that $t_{p} \leq t_{\sigma} \leq$ $T 6$ See Figure 2 for a graphical representation of the tight bound. Achievability for all cases will be demonstrated by efficient protocols that neither rely on any particular signature scheme nor on any particular way of setting up a PKI.

As an interesting special case, the optimal result of [GMW87] (assuming a consistent PKI instead of broadcast - allowing to drop parameter $t_{p}$ since the consistency of the PKI is granted) computationally secure against $T<n / 2$ corrupted players additionally allows to guarantee unconditional security against $t_{\sigma} \leq n / 4$ corrupted players "for free." On the other hand, when requiring optimality with respect to unconditional security, $t_{\sigma}=\lfloor(n-1) / 3\rfloor$, then practically no higher computational bound $T$ can be simultaneously tolerated on top.

Finally, when basing the PKI on an unconditional pseudo-signature scheme (which is not our focus), forgery becomes impossible by definition and the tight bound collapses to $2 T+t_{p}<n$.

Constructions. Our final MPC protocol is obtained by simulating broadcast in the unconditional MPC protocol of [CDD ${ }^{+} 99$. Thus the main technical contribution in this paper is to simulate broadcast (aka Byzantine agreement) in the given models with respect to the required security aspects. The (efficient) protocol in $\left[\mathrm{CDD}^{+} 99\right]$ is unconditionally secure against $t<n / 2$ corrupted players. So, obviously, the final MPC protocol wherein broadcast is simulated is as secure as the given broadcast protocol.

${ }^{6}$ That is, additional forgery gives the adversary no additional power when the PKI is inconsistent. 


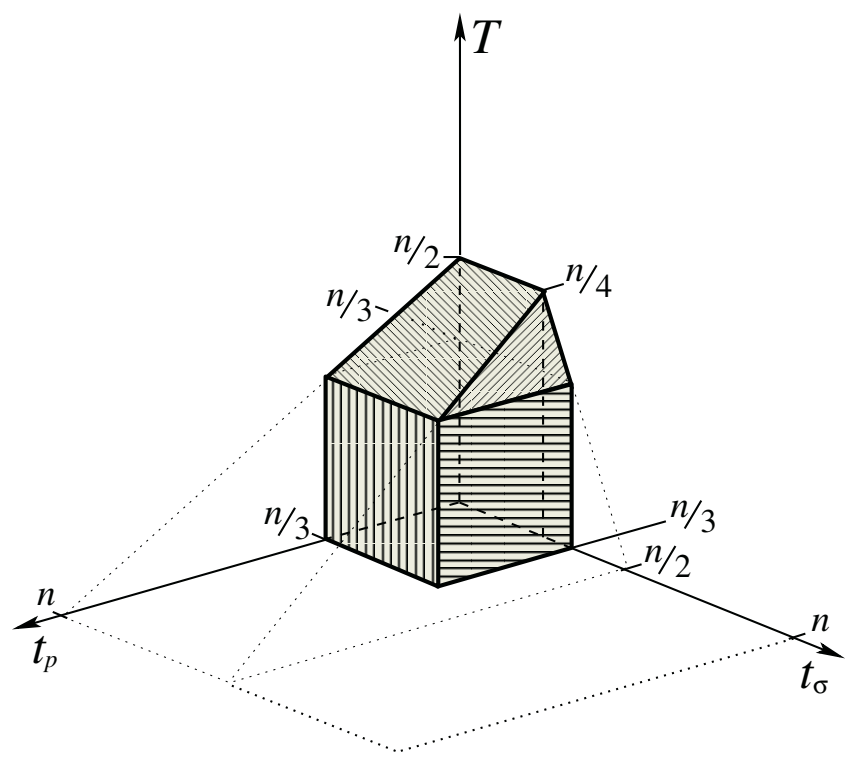

Fig. 2. Graphical representation of Bound (1).

\section{Definitions and Notations}

\subsection{Multi-party Computation}

In MPC a set of players want to distributedly evaluate some agreed function(s) on their inputs in a way preserving privacy of their inputs and correctness of the computed result. More precisely, in an MPC among a player set $P$ with respect to a collection of functions $\left(f_{1}, \ldots, f_{n}\right)$, every player $p_{i} \in P$ holds a secret input (vector) $x_{i}$ and secretly receives an output (vector) $y_{i}=f_{i}\left(x_{1}, \ldots, x_{n}\right)$.

From a qualitative point of view, the security of MPC is often broken down to the conditions "privacy", "correctness", "robustness", and "fairness", and ideally, a protocol should satisfy all these properties.

PrIVACY. A protocol achieves privacy if the adversary cannot learn more about the correct players' inputs than given by the inputs and outputs of the corrupted players.

CorreCtness. A protocol achieves correctness if the correct players' outputs are indeed computed as defined by the functions $f_{i}$.

Robustness. A protocol achieves robustness if every correct player finally receives his outputs.

FAIRNESS. A protocol achieves fairness if the adversary gets no information about the correct players' inputs in case that robustness is not achieved.

More formally, MPC is modeled by an ideal process involving a mutually trusted party $\tau$ where the players secretly hand their inputs to $\tau$, followed by 
$\tau$ computing the players' outputs and secretly handing them back to the corresponding players Bea91, Can00, Gol01. This model is referred to as the ideal model The goal of MPC is now to achieve the same functionality in the so-called real model where there is no such trusted party such that an adversary gets no advantage compared to an execution of the ideal protocol. An MPC protocol is defined to be secure if, for every adversary $\mathcal{A}$ in the protocol, there is an adversary $\mathcal{S}$ in the ideal model that, with similar costs, achieves (essentially) the same output distribution as the adversary in the protocol [Bea91, Can00, Gol01].

\subsection{Broadcast}

Broadcast is the special case of an MPC. In broadcast, one player $p_{s}$ is given an initial value which everybody is required to receive. The definition is as follows:

Definition 1 (Broadcast). A protocol among $n$ players, where player $p_{s} \in P$ (called the sender) holds an input value $x_{s}$ and every player $p_{i}(i \in\{1, \ldots, n\})$ computes an output value $y_{i}$, achieves broadcast if it satisfies:

- VALIDITY: If the sender is correct then all correct players $p_{i}$ compute output $y_{i}=x_{s}$.

- Consistency: All correct players compute the same output value $y$.

Often, it is also added to the definition that the protocol is always required to terminate. We will not mention this property explicitly since termination is obvious for our protocols.

Note that broadcast for any finite domain easily reduces to binary broadcast (where the sender sends a value from $\{0,1\}$ ) as, e.g., shown by Turpin and Coan [TC84]. We will thus focus on binary broadcast.

During the simulation of broadcast, using signatures, it must be avoided that previous signatures can be reused by the adversary in a different context, i.e., an independent phase or another instance of the protocol. This fact was observed in [GLR95] and more profoundly treated in [LLR02]. To avoid such "replay attacks" values can be combined with unique sequence numbers before signing. The sequence numbers themselves do not have to be transferred since they can be generated in a predefined manner (encoding the protocol instance and the communication round). However, we will not explicitly state these details in the descriptions of the sequel.

\section{$2.3 \quad$ Setting}

We consider a set $P=\left\{p_{1}, \ldots, p_{n}\right\}$ of $n$ players that are connected via a complete synchronous network of pairwise secure channels. We assume a PKI to be set up among the players.

A given PKI is consistent if every player $p_{i}(i \in\{1, \ldots, n\})$ has a secretkey/public-key pair $\left(S K_{i}, P K_{i}\right)$ which was chosen by $p_{i}$ with respect to the keygeneration algorithm of a digital signature scheme and, additionally, that each 
respective public key $P K_{i}$ is known to all players as $p_{i}$ 's public key to be exclusively used for the verification of $p_{i}$ 's signatures. Asserting that, with respect to every signer $p_{i}$, each player holds the same public key $P K_{i}$ guarantees that $p_{i}$ 's signatures can be transferred between the players without losing conclusiveness. In our model, the PKI may or may not be consistent 7

We assume the existence of an active threshold adversary to corrupt some of the players. The adversary may or may not be able to forge signatures. Furthermore, for our protocols, the adversary is assumed to be adaptive (but nonmobile). In contrast, our proofs of optimality even hold with respect to a static adversary. As we have three bounds with respect to player corruption, $t_{p}, t_{\sigma}$, and $T$, we will make a stringent distinction between these bounds and the actual number of players corrupted at the end of the protocol, which we will denote by $f 8$

\subsection{Protocol Notation}

Protocols are specified with respect to a player set $P$ and stated with respect to the local view of player $p_{i}$, meaning that all players $p_{i} \in P$ execute this code in parallel with respect to their own identity $i$.

With respect to pairwise communication we also consider reflexive channels among the players for simplicity. Thus, when a player $p_{i}$ sends a value to each player then $p_{i}$ also receives a copy himself.

For simplicity, in our protocol notation, we do not explicitly state how to handle values received from corrupted players that are outside the specified domain. Such a value is always implicitly assumed to be replaced by a default value or by any arbitrary value inside the specified domain.

\section{Generic Broadcast Simulation for $\mathrm{t}<\mathrm{n} / 2$}

Our broadcast simulations are based on the "phase king" protocol in BGP89. In FM00, it was observed that any protocol for "weak broadcast" is sufficient in order to achieve broadcast secure against a faulty minority — as secure as the given protocol for weak broadcast. Since all of our tight bounds imply that strictly less than half of all players are corrupted we thus only need to give respective protocols for weak broadcast.

Weak broadcast (as called in FM00]) was originally introduced in [Dol82] under the name crusader agreement. Weak broadcast is the same as broadcast for the case that the sender is correct but, if the sender is corrupted, then some players might end up with the "invalidity symbol" $\perp$ - but still, it guarantees that no two correct players end up with two different values in $\{0,1\}$.

\footnotetext{
${ }^{7}$ In case of unconditional pseudo-signatures the situation is slightly different since, instead of the same public key $P K_{i}$, each player $p_{j}$ holds a different "public key" $P K_{i j}$ (which is in fact secret).

${ }^{8}$ As the adversary is adaptive, the number might increase during the execution of the protocol, and reach its maximum at the end.
} 
Definition 2 (Weak broadcast). A protocol where one player $p_{s}$ has an input $x_{s} \in\{0,1\}$ and every player $p_{i}$ computes an output $y_{i} \in\{0,1, \perp\}$ achieves weak broadcast if it satisfies the following conditions:

- VALIDITY: If $p_{s}$ is correct then every correct player $p_{i}$ computes output $y_{i}=$ $x_{s}$.

- Consistency: If player $p_{i}$ is correct and computes $y_{i} \in\{0,1\}$ then every correct player $p_{j}$ computes $y_{j} \in\left\{y_{i}, \perp\right\}$.

In Appendix A, we describe a reduction from broadcast to weak broadcast that is simpler and more efficient than the one in [FM00], yielding the following theorem.

Theorem 1. If at most $t<n / 2$ players are corrupted then efficient achievability of weak broadcast implies efficient achievability of broadcast.

\section{Tight Bounds}

We now demonstrate the tightness of the bound given in Bound (1).

\subsection{Efficient Protocol}

We first give an efficient protocol for broadcast and then show how to plug it into the MPC protocol in $\left[\mathrm{CDD}^{+} 99\right]$ in order to get out final protocol for efficient hybrid MPC.

Broadcast. For the constructive part, according to Theorem 1, it is sufficient to give a construction for weak broadcast. The following protocol is designed for any selection of thresholds $t_{p}, t_{\sigma}$, and $T,\left(t_{p} \leq t_{\sigma} \leq T\right)$, satisfying Bound (1).

Let $x_{s}$ be $p_{s}$ 's input value, and let $\sigma_{s}\left(x_{s}\right)$ be a signature by $p_{s}$ on the value $x_{s}$. Furthermore, let $V$ be the signature verification algorithm with respect to the underlying signature scheme computing $V(x, \sigma, \mathrm{PK})=1$ if $\sigma$ is a valid signature on $x$ with respect to public key $\mathrm{PK}$, and $V(x, \sigma, \mathrm{PK})=0$ otherwise. Let $\mathrm{PK}_{i}^{s}$ be player $p_{i}$ 's version of $p_{s}$ 's public key. We use $V_{i}^{s}(x, \sigma)$ as a short cut for $V\left(x, \sigma, \mathrm{PK}_{i}^{s}\right)$. With respect to player $p_{i}$, we say that a given signature $\sigma$ is valid if it is valid with respect to $p_{i}$ 's view, i.e., $V_{i}^{s}(x, \sigma)=1$. In particular, a valid signature with respect to player $p_{i}$ 's view might in fact not have been issued by the respective signer.

The protocol works as follows. The sender $p_{s}$ signs his input value and sends his input together with its signature to every other player: $\left(x_{s}, \sigma_{s}\left(x_{s}\right)\right)$. Every player except for the sender now redistributes this information to everybody (but without signing this new message himself). Now, every player received $n$ values, one from every player. Each player $p_{i}$ now decides on the outcome of the protocol:

- Let $x_{i}^{s}$ be the bit he directly received from the sender. If the bit $x_{i}^{s}$ is received from at least $n-t_{p}$ different players overall then he computes output $y_{i}=x_{i}$. 
- Otherwise, if he received the bit $x_{i}^{s}$ together with a valid signature by $p_{s}$ from the sender and at least $n-t_{\sigma}$ different players overall then he decides on $y_{i}=x_{i}$.

- Otherwise, if he received the bit $x_{i}$ together with a valid signature by $p_{s}$ from the sender and at least $n-T$ different players overall — but no single correct signature by $p_{s}$ for bit $1-x_{i}$ - then he decides on $y_{i}=x_{i}$.

- Otherwise, he decides on $y_{i}=\perp$.

Protocol 1 WeakBroadcast $p_{s}\left(P, x_{s}\right)$

1. if $i=s$ then SendToAll $\left(x_{s}, \sigma_{s}\left(x_{s}\right)\right) \mathrm{fi} ; \quad \operatorname{Receive}\left(x_{i}^{s}, \sigma_{i}^{s}\right)$;

2. if $i \neq s$ then $\operatorname{SendToAll}\left(x_{i}^{s}, \sigma_{i}^{s}\right)$ fi; $\quad \forall j \neq s$ : Receive $\left(x_{i}^{j}, \sigma_{i}^{j}\right)$;

3. $U_{i}^{0}:=\left\{p_{j} \in P \mid x_{i}^{j}=0\right\} ; U_{i}^{1}:=\left\{p_{j} \in P \mid x_{i}^{j}=1\right\}$;

4. $S_{i}^{0}:=\left\{p_{j} \in P \mid x_{i}^{j}=0 \wedge V_{i}^{s}\left(0, \sigma_{i}^{j}\right)=1\right\}$;

$S_{i}^{1}:=\left\{p_{j} \in P \mid x_{i}^{j}=1 \wedge V_{i}^{s}\left(1, \sigma_{i}^{j}\right)=1\right\}$;

5. if $\left|U_{i}^{x_{i}^{s}}\right| \geq n-t_{p}$ then $y_{i}:=x_{i}^{s}$

elseif $p_{s} \in S_{i}^{x_{i}^{s}} \wedge\left|S_{i}^{x_{i}^{s}}\right| \geq n-t_{\sigma}$ then $y_{i}:=x_{i}^{s}$

elseif $p_{s} \in S_{i}^{x_{i}^{s}} \wedge\left|S_{i}^{x_{i}^{s}}\right| \geq n-T \wedge S_{i}^{1-x_{i}^{s}}=\emptyset$ then $y_{i}:=x_{i}^{s}$

else $y_{i}:=\perp \mathrm{fi}$;

6. return $y_{i}$

Lemma 1 (Weak Broadcast). Protocol 1 among the players $P=\left\{p_{1}, \ldots, p_{n}\right\}$ achieves efficient weak broadcast with sender $p_{s} \in P$ if $2 T+t_{p}<n$ and $T+2 t_{\sigma}<$ $n$.

Proof. We show that the validity and consistency properties are satisfied. For this, let $f$ be the number of corrupted players at the end of the protocol. Efficiency is obvious.

VALIDITY: Suppose that the sender $p_{s}$ is correct. Hence, every correct player $p_{i}$ receives the sender's input $x_{s}$ during Step 1 of the protocol, $x_{i}^{s}=x_{s}$, and a signature $\sigma_{i}^{s}$.

If $f \leq t_{p}$ then every correct player $p_{i}$ receives the value $x_{s}$ from at least $n-t_{p}$ different players (including the sender) during Steps 1 and 2. Hence, $\left|U_{i}^{x_{s}}\right| \geq n-t_{p}$, and $p_{i}$ computes $y_{i}=x_{s}$ according to Condition (A) in Step 5.

If $t_{p}<f \leq t_{\sigma}$ then every correct player $p_{i}$ receives the value $x_{s}$ together with a valid signature by $p_{s}$ (note that the PKI is consistent in this case) from at least $n-t_{\sigma}$ different players (including the sender) during Steps 1 and 2 . Hence, $\left|S_{i}^{x_{s}}\right| \geq n-t_{\sigma}$, and $p_{i}$ computes $y_{i}=x_{s}$ according to Conditions (A) or (B) in Step 5 .

If $t_{\sigma}<f \leq T$ then every correct player $p_{i}$ receives the value $x_{s}$ together with a valid signature by $p_{s}$ from at least $n-T$ different players (including the sender) during Steps 1 and 2. Hence, $\left|S_{i}^{x_{s}}\right| \geq n-T$ and $S_{i}^{1-x_{s}}=\emptyset$ since the adversary cannot forge signatures in this case. Hence, $p_{i}$ computes $y_{i}=x_{s}$ according to Conditions (A), (B), or (C). 
Consistency: Suppose that some correct player $p_{i}$ computes output $y_{i} \neq \perp$. We have to show that hence, every correct player $p_{j}$ computes an output $y_{j} \in\left\{y_{i}, \perp\right\}$.

Suppose first, that $p_{i}$ decides according to Condition (A) in Step 5, i.e., $\left|U_{i}^{y_{i}}\right| \geq n-t_{p}$. For $p_{j}$ this implies that $\left|U_{j}^{y_{i}}\right| \geq\left|U_{i}^{y_{i}}\right|-T \geq n-t_{p}-T>T$ and hence that $\left|S_{j}^{1-y_{i}}\right| \leq\left|U_{j}^{1-y_{i}}\right|<n-T$ and thus that $p_{j}$ cannot compute $y_{j}=1-y_{i}$, neither according to Conditions (A), (B), nor (C).

Second, suppose that $p_{i}$ decides according to Condition (B) in Step 5, i.e., $\left|S_{i}^{y_{i}}\right| \geq n-t_{\sigma}$. It remains to show that $p_{j}$ does not decide on $y_{j}=1-y_{i}$ according to Conditions (B) or (C) (the rest is out-ruled by the last paragraph). For $p_{j}$ the assumption implies that

$$
\left|U_{j}^{y_{i}}\right| \geq\left|U_{i}^{y_{i}}\right|-f \geq n-t_{\sigma}-f \geq\left\{\begin{array}{ll}
n-2 t_{\sigma}>T & , \text { if } f \leq t_{\sigma} \\
n-t_{\sigma}-T>t_{\sigma} & , \text { if } f \leq T
\end{array} .\right.
$$

Now, if $f \leq t_{\sigma}$ then $\left|S_{j}^{1-y_{i}}\right| \leq\left|U_{j}^{1-y_{i}}\right|<n-T$, and $p_{j}$ cannot compute $y_{j}=1-y_{i}$ according to Conditions (B) or (C). If $t_{\sigma}<f \leq T$ then $\left|U_{j}^{1-y_{i}}\right|<n-t_{\sigma}$, and $S_{j}^{y_{i}} \neq \emptyset$ (since the PKI is consistent and $p_{i}$ holds and redistributes a valid signature on $y_{i}$ ), and thus $p_{j}$ still cannot compute $y_{j}=1-y_{i}$ according to Conditions (B) or (C).

Third, suppose that $p_{i}$ decides according to Condition (C) in Step 5, i.e., $\left|S_{i}^{y_{i}}\right| \geq n-T$ and $S_{i}^{1-y_{i}}=\emptyset$. It remains to show that $p_{j}$ cannot decide on $y_{j}=1-y_{i}$ according to Condition (C). Now, $f \leq t_{p}$ implies $\left|U_{j}^{1-y_{i}}\right|<n-T$ (since $\left|U_{j}^{y_{i}}\right| \geq n-T-t_{p}>T$ ), and $f>t_{p}$ implies $S_{j}^{y_{i}} \neq \emptyset$ (since the PKI is consistent). Finally, both implications rule out that $p_{j}$ computes $y_{j}=1-y_{i}$ according to Condition (C).

Multi-party Computation. The MPC protocol in $\left[\mathrm{CDD}^{+99}\right]$ unconditionally tolerates an (adaptive) adversary that corrupts up to $t<n / 2$ players — but assuming broadcast channels to be available.

Theorem 2. Hybrid MPC is efficiently achievable if $2 T+t_{p}<n$ and $T+2 t_{\sigma}<$ $n$.

Proof. Efficient achievability of hybrid broadcast for $2 T+t_{p}<n$ and $T+2 t_{\sigma}<n$ follows from Lemma 1 and Theorem 1 We can now simulate each invocation of a broadcast channel in $\mathrm{CDD}^{+99}$ with an instance of such a hybrid broadcast protocol. Since Bound (11) implies $2 T<n$, we have that $t_{p} \leq t_{\sigma} \leq T<n / 2$. Thus, an adversary that is tolerated in the broadcast protocol is automatically tolerated in the MPC protocol.

\subsection{Tightness}

We now show that Bound (II) is tight. We do this in three steps. First, we show that hybrid broadcast is impossible if $t_{p}>0, t_{\sigma}=0$, and $2 T+t_{p} \geq n$. Second, we show that hybrid broadcast is impossible if $t_{p}=0, t_{\sigma}>0$, and $T+2 t_{\sigma} \geq n$. Third, we use the fact that MPC is impossible whenever $2 T \geq n$ [Cle86]. 
Impossibility of Broadcast for $t_{p}+2 T \geq n$ when $t_{p}>0$. The proof proceeds along the lines of the proof in [FLM86] that unconditional broadcast for $t \geq n / 3$ is impossible. The idea is to assume any protocol among $n$ players that (possibly) achieves broadcast for $n \leq 2 T+t_{p}\left(t_{p}>0, t_{\sigma}=0\right)$ and to use it to build a different distributed system whose behavior demonstrates that the original protocol must be insecure. It is important to note that this new system is not required to achieve broadcast. It is simply a distributed system whose behavior is determined by the protocol or, more precisely, by the corresponding local programs of the involved players. Also, it is assumed that no adversary is present in this system. Rather, with respect to some of the players, the way the new system is composed simulates an admissible adversary with respect to these players in the original system. Thus, with respect to these players, all conditions of broadcast are required to be satisfied among them even in this new system. Finally, it is shown that all of these players' respective conditions cannot be satisfied simultaneously and thus that the protocol cannot achieve broadcast.

Building the new system. Assume any protocol $\Psi$ for a player set $P$ with sender $p_{0}$ and $|P|=n \geq 3$ that tolerates $2 T+t_{p} \geq n$ (with $t_{p}>0$ ).

Let $\Pi=\left\{\pi_{0}, \ldots, \pi_{n-1}\right\}$ be the set of the players' corresponding processors with their local programs sharing a consistent PKI where player $p_{i}$ 's secretkey/public-key pair is $\left(\mathrm{SK}_{i}, \mathrm{PK}_{i}\right)$ and player $p_{j}$ 's copy of the respective public key is $\mathrm{PK}_{i j}$. Since $0<t_{p} \leq T$, it is possible to partition the processors into three sets, $\Pi_{0} \dot{\cup} \Pi_{1} \dot{\cup} \Pi_{2}=\Pi$, such that $1 \leq\left|\Pi_{0}\right| \leq t_{p}, 1 \leq\left|\Pi_{1}\right| \leq T$, and $1 \leq\left|\Pi_{2}\right| \leq T$.

For each $\pi_{i} \in \Pi_{0}$, let $\pi_{i}^{\prime}$ be an identical copy of processor $\pi_{i}$. Let the number $i$ denote the type of any processor $\pi_{i}$ (or $\pi_{i}^{\prime}$, respectively). Furthermore, let $\Pi_{0}^{\prime}=\left\{\pi_{i}^{\prime} \mid \pi_{i} \in \Pi_{0}\right\}$ form an identical copy of set $\Pi_{0}$. For all $\pi_{i} \in \Pi_{0}^{\prime}$, generate a new secret-key/public-key pair $\left(\mathrm{SK}_{i}^{\prime}, \mathrm{PK}_{i}^{\prime}\right)$ and overwrite $\pi_{i}$ 's own secret key $\mathrm{SK}_{i}:=\mathrm{SK}_{i}^{\prime}$. Additionally, for all $\pi_{j} \in \Pi_{2} \cup \Pi_{0}^{\prime}$, overwrite $\pi_{j}$ 's copy of $\pi_{i}$ 's public key: $\mathrm{PK}_{i j}:=\mathrm{PK}_{i}^{\prime}$ (and $\mathrm{PK}_{i}:=\mathrm{PK}_{i}^{\prime}$ ). See Figure 3 .

Instead of connecting the original processors as required for broadcast, we build a network involving all processors in $\Pi_{0} \cup \Pi_{1} \cup \Pi_{2} \cup \Pi_{0}^{\prime}$ with their pairwise communication channels connected in a way such that each processor $\pi_{i}$ (or $\pi_{i}^{\prime}$ ) communicates with at most one processor of each type $j \in\{1, \ldots, n\} \backslash\{i\}$.

Consider Figure 3 Exactly all pairs in $\left(\Pi_{0} \cup \Pi_{1}\right) \times\left(\Pi_{0} \cup \Pi_{1}\right),\left(\Pi_{1} \cup \Pi_{2}\right) \times\left(\Pi_{1} \cup\right.$ $\left.\Pi_{2}\right)$, and $\left(\Pi_{2} \cup \Pi_{0}^{\prime}\right) \times\left(\Pi_{2} \cup \Pi_{0}^{\prime}\right)$ are connected by pairwise channels. There are no connections between the sets $\Pi_{0}$ and $\Pi_{2}$, and no connections between the sets $\Pi_{1}$ and $\Pi_{0}^{\prime}$. Messages that originally would have been sent from a processor in $\Pi_{0}$ to a processor in $\Pi_{2}$ are discarded. Messages that originally would have been sent from a processor in $\Pi_{2}$ to a processor in $\Pi_{0}$ are delivered to the corresponding processor in $\Pi_{0}^{\prime}$. Messages sent from a processor in $\Pi_{0}^{\prime}$ to a processor in $\Pi_{1}$ are discarded.

We now show that for the sets $\Pi_{0} \cup \Pi_{1}, \Pi_{1} \cup \Pi_{2}$, and $\Pi_{2} \cup \Pi_{0}^{\prime}$, and for inputs $x_{0}=0$ and $x_{0}^{\prime}=1$, each set's joint view is indistinguishable from its view in the original setting for an adversary corrupting the remaining processors in 


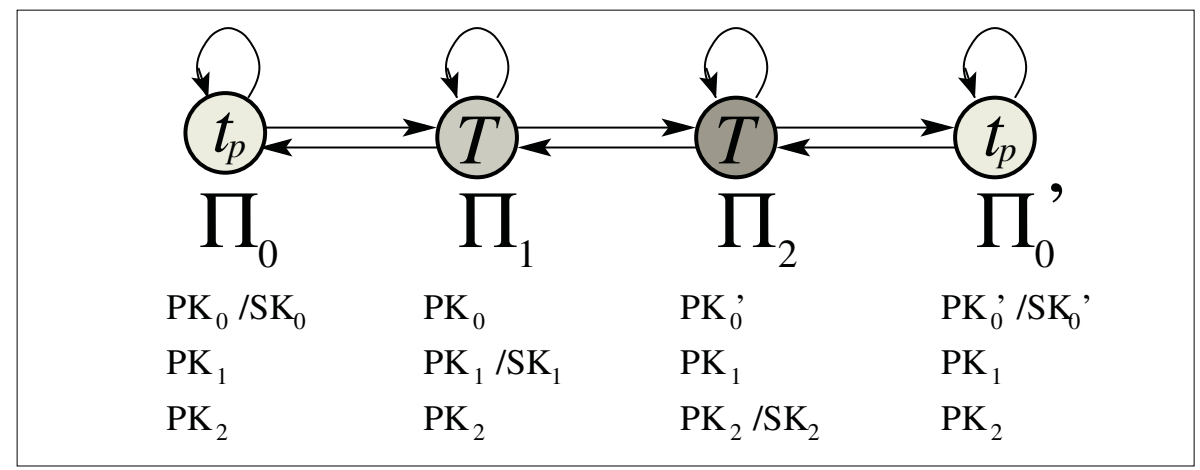

Fig. 3. Rearrangement of processors in proof of Theorem[3.

an admissible way, and possibly have made for a bad PKI if it corrupts at most $f \leq t_{p}$ processors.

Lemma 2. If the input of $\pi_{0}$ is $x_{0}=0$ then the joint view of the processors in $\Pi_{0} \cup \Pi_{1}$ is indistinguishable from their view in the original system when the adversary corrupts the processors in $\Pi_{2}$ in an admissible way.

Proof. By corrupting all processors in $\Pi_{2}$ in the original system the adversary simulates all processors in $\Pi_{2} \cup \Pi_{0}^{\prime}$ of the new system. For all $\pi_{i} \in \Pi_{0}^{\prime}$ it generates a new secret-key/public-key pair $\left(\mathrm{SK}_{i}^{\prime}, \mathrm{PK}_{i}^{\prime}\right)$ and overwrites $\pi_{i}$ 's own secret key $\mathrm{SK}_{i}:=\mathrm{SK}_{i}^{\prime}$ and, for all $\pi_{j} \in \Pi_{2} \cup \Pi_{0}^{\prime}$, overwrites $\pi_{j}$ 's copy of $\pi_{i}$ 's public key: $\mathrm{PK}_{i j}:=\mathrm{PK}_{i}^{\prime}$ (and $\mathrm{PK}_{i}:=\mathrm{PK}_{i}^{\prime}$ ). Initially, the adversary overwrites input $x_{0}^{\prime}:=1$. The PKI among the processors in $\Pi_{0} \cup \Pi_{1}$ is still fully consistent and thus the joint view of the processors in $\Pi_{0} \cup \Pi_{1}$ in the original system is exactly the same as their view in the new system.

Lemma 3. If the input of $\pi_{0}^{\prime}$ is $x_{0}^{\prime}=1$ then the joint view of the processors in $\Pi_{2} \cup \Pi_{0}^{\prime}$ is indistinguishable from their view in the original system when the adversary corrupts the processors in $\Pi_{1}$ in an admissible way.

Proof. By symmetry, this case follows from Lemma $2^{9}$

Lemma 4. The joint view of the processors in $\Pi_{1} \cup \Pi_{2}$ is indistinguishable from their view in the original system when the adversary corrupts the processors in $\Pi_{0}$ in an admissible way.

${ }^{9}$ The only difference in this case is that $\Pi_{0}^{\prime}$ takes the role of the original set and $\Pi_{0}$ the role of its copy. Accordingly, the initial key pairs are $\left(\mathrm{SK}_{i}^{\prime}, \mathrm{PK}_{i}^{\prime}\right)$, the pairs $\left(\mathrm{SK}_{i}, \mathrm{PK}_{i}\right)$ are newly generated by the adversary, and $x_{0}:=0$ is overwritten. 
Proof. Since $\left|\Pi_{0}\right| \leq t_{p}$ the adversary can have previously made the PKI inconsistent by generating and respectively distributing the key pairs $\left(\mathrm{SK}_{i}^{\prime}, \mathrm{PK}_{i}^{\prime}\right)$ for all $\pi_{i} \in \Pi_{0}^{\prime}$ (according to Figure 3). By corrupting all processors in $\Pi_{0}$ in the original system the adversary can now simulate all processors in $\Pi_{0} \cup \Pi_{0}^{\prime}$ of the new system whereas, initially, it overwrites $x_{0}:=0$ and $x_{0}^{\prime}:=1$. Thus the joint view of the processors in $\Pi_{1} \cup \Pi_{2}$ in the original system is exactly the same as their view in the new system.

Theorem 3. If $2 T+t_{p} \geq n$ and $t_{p}>0$ then there exists no hybrid broadcast protocol. In particular, for every protocol there exists a sender input $x_{0} \in\{0,1\}$ such that a computationally bounded adversary can make the protocol fail with some non-negligible probability - by either corrupting $T$ players, or by corrupting $t_{p}$ players and additionally having made for an inconsistent PKI.

Proof. Assume that $x_{0}=0$ and $x_{0}^{\prime}=1$. Then, by Lemmas 2, 3, and 4 each mentioned set's joint view in the new system is indistinguishable from their view in the original system. However, for each run of the new system, either validity is violated among the processors in $S_{01}=\Pi_{0} \cup \Pi_{1}$ or $S_{20^{\prime}}=\Pi_{2} \cup \Pi_{0}^{\prime}$, or consistency is violated among the processors in $S_{12}=\Pi_{1} \cup \Pi_{2}$.

Thus there is a sender input $\left(x_{0}=0\right.$ or $\left.x_{0}^{\prime}=1\right)$ such that the adversary can make the protocol fail with non-negligible probability by uniformly randomly choosing a processor set $\Pi_{i}$ and corrupting the respective processors correspondingly.

Impossibility of Broadcast for $2 \mathrm{t}_{\sigma}+\mathbf{T} \geq \mathbf{n}$ when $\mathrm{t}_{\sigma}>\mathbf{0}$. The proof of this case is very similar to the proof of Theorem 3

Theorem 4. If $T+2 t_{\sigma} \geq n$ and $t_{\sigma}>0$ then there exists no hybrid broadcast protocol. In particular, for every protocol there exists a sender input $x_{0} \in\{0,1\}$ such that the adversary can make the protocol fail with some non-negligible probability - by either corrupting $T$ players, or by corrupting $t_{\sigma}$ players and additionally being able to forge signatures with non-negligible probability.

Proof. Assume any protocol $\Psi$ for a player set $P$ with sender $p_{0}$ and $|P|=n \geq 3$ that tolerates $2 T+t_{\sigma} \geq n$ (with $t_{\sigma}>0$ ).

Let $\Pi=\left\{\pi_{0}, \ldots, \pi_{n-1}\right\}$ be the set of the players' corresponding processors with their local programs. Since $0<t_{\sigma} \leq T$, it is possible to partition the processors into three sets, $\Pi_{0} \dot{\cup} \Pi_{1} \cup \dot{\cup} \Pi_{2}=\Pi$, such that $1 \leq\left|\Pi_{0}\right| \leq T, 1 \leq\left|\Pi_{1}\right| \leq$ $t_{\sigma}$, and $1 \leq\left|\Pi_{2}\right| \leq t_{\sigma}$.

For each $\pi_{i} \in \Pi_{0}$, let $\pi_{i}^{\prime}$ be an identical copy of processor $\pi_{i}$ and, as in the proof of Theorem 3, let $\Pi_{0}^{\prime}=\left\{\pi_{i}^{\prime} \mid \pi_{i} \in \Pi_{0}\right\}$ form an identical copy of set $\Pi_{0}$.

Consider Figure 4 Exactly all pairs in $\left(\Pi_{0} \cup \Pi_{1}\right) \times\left(\Pi_{0} \cup \Pi_{1}\right),\left(\Pi_{1} \cup \Pi_{2}\right) \times$ $\left(\Pi_{1} \cup \Pi_{2}\right)$, and $\left(\Pi_{2} \cup \Pi_{0}^{\prime}\right) \times\left(\Pi_{2} \cup \Pi_{0}^{\prime}\right)$ are connected by pairwise channels.

Again, we show that for the sets $\Pi_{0} \cup \Pi_{1}, \Pi_{1} \cup \Pi_{2}$, and $\Pi_{2} \cup \Pi_{0}^{\prime}$, and for inputs $x_{0}=0$ and $x_{0}^{\prime}=1$, each set's joint view is indistinguishable from its view in the original setting. 


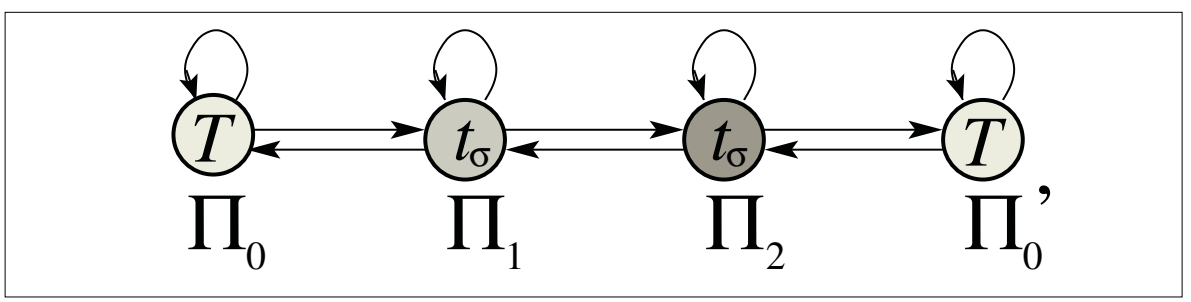

Fig. 4. Rearrangement of processors in proof of Theorem 3 .

Joint view of $\Pi_{0} \cup \Pi_{1}$ with $x_{0}=0$. By corrupting all processors in $\Pi_{2}$ in the original system the adversary simulates all processors in $\Pi_{2} \cup \Pi_{0}^{\prime}$ of the new system. Since $\left|\Pi_{2}\right| \leq t_{\sigma}$, the adversary can forge all signatures by processors in $\Pi_{0}^{\prime}$ required for the simulation. Initially, the adversary overwrites input $x_{0}^{\prime}:=1$. Thus the joint view of the processors in $\Pi_{0} \cup \Pi_{1}$ in the original system is exactly the same as their view in the new system.

Joint view of $\Pi_{2} \cup \Pi_{0}^{\prime}$ with $x_{0}^{\prime}=1$. By symmetry, this case follows from the above paragraph.

Joint view of $\Pi_{1} \cup \Pi_{2}$. By corrupting all processors in $\Pi_{0}$ in the original system the adversary can simulate all processors in $\Pi_{0} \cup \Pi_{0}^{\prime}$ of the new system whereas, initially, it overwrites $x_{0}:=0$ and $x_{0}^{\prime}:=1$. Note that, by corrupting the processors in $\Pi_{0}$, the adversary gains access to all corresponding secret keys and thus is not required to forge any signatures for the simulation. Thus the joint view of the processors in $\Pi_{1} \cup \Pi_{2}$ in the original system is exactly the same as their view in the new system.

The theorem now follows along the lines of the proof of Theorem 3 .

Multi-party Computation. In order to complete our tightness argument, we require the following proposition.

Proposition 1 ([Cle86]). There is no protocol for $M P C$ secure against $T \geq$ $n / 2$ actively corrupted players. In particular, fairness cannot be guaranteed.

Theorem 5. Hybrid MPC is impossible if either $2 T+t_{p} \geq n$ or $T+2 t_{\sigma} \geq n$.

Proof. The theorem follows from Theorems 3 and 4 , and Proposition 1 .

\section{Conclusion and Open Problems}

We can now conclude tight bounds for the achievability of hybrid MPC with respect to thresholds $t_{p}, t_{\sigma}$, and $T$. 
Theorem 6. Hybrid MPC is (efficiently) achievable if and only if $2 T+t_{p}<n$ and $T+2 t_{\sigma}<n$.

Proof. The theorem follows from Theorems 2 and 5.

In particular, assuming the PKI to be consistent in any case (as in the alternative model for GMW87] assuming a PKI instead of broadcast) we can drop parameter $t_{p}$ and immediately get the tight bound $2 T<n \wedge T+2 t_{\sigma}<n$. This means that, with respect to this model, computational security against $f \leq T<n / 2$ corrupted players can be combined with unconditional security against $f \leq t_{\sigma}$ corrupted players.

The characterization given in Theorem[6] is tight with respect to fully secure, robust MPC. However, as mentioned in the introduction, non-robust MPC is also possible in presence of a corrupted majority. Thus, for the case $t_{p}=0$, it remains an open question whether hybrid non-robust MPC can be achieved for any $T+2 t_{\sigma}<n$.

\section{References}

[Bea89] Donald Beaver. Multiparty protocols tolerating half faulty processors. In Advances in Cryptology: CRYPTO '89, volume 435 of Lecture Notes in Computer Science, pages 560-572. Springer-Verlag, 1989.

[Bea91] Donald Beaver. Foundations of secure interactive computation. In $A d$ vances in Cryptology: CRYPTO '91, volume 576 of Lecture Notes in Computer Science, pages 377-391. Springer-Verlag, 1991.

[BG89] Donald Beaver and Shafi Goldwasser. Multiparty computation with faulty majority. In Proceedings of the 30th Annual IEEE Symposium on Foundations of Computer Science (FOCS '89), pages 468-473, 1989.

[BGP89] Piotr Berman, Juan A. Garay, and Kenneth J. Perry. Towards optimal distributed consensus (extended abstract). In Proceedings of the 30th Annual IEEE Symposium on Foundations of Computer Science (FOCS '89), pages 410-415, 1989.

[BGW88] Michael Ben-Or, Shafi Goldwasser, and Avi Wigderson. Completeness theorems for non-cryptographic fault-tolerant distributed computation. In Proceedings of the 20th Annual ACM Symposium on Theory of Computing (STOC '88), pages 1-10. Springer-Verlag, 1988.

[Can00] Ran Canetti. Security and composition of multiparty cryptographic protocols. Journal of Cryptology, 13(1):143-202, 2000.

[CCD88] David Chaum, Claude Crépeau, and Ivan Damgård. Multiparty unconditionally secure protocols (extended abstract). In Proceedings of the 20th Annual ACM Symposium on Theory of Computing (STOC '88), pages 11-19. ACM Press, 1988.

[CDD ${ }^{+99}$ Ronald Cramer, Ivan Damgård, Stefan Dziembowski, Martin Hirt, and Tal Rabin. Efficient multiparty computations secure against an adaptive adversary. In Advances in Cryptology: EUROCRYPT '99, volume 1592 of Lecture Notes in Computer Science, 1999.

[Cha89] David Chaum. The spymasters double-agent problem. In Advances in Cryptology: CRYPTO '89, volume 435 of Lecture Notes in Computer Science, pages 591-602. Springer-Verlag, 1989. 
[Cle86] Richard Cleve. Limits on the security of coin flips when half the processors are faulty. In ACM Symposium on Theory of Computing (STOC '86), pages 364-369, Baltimore, USA, May 1986. ACM Press.

$\left[\mathrm{DFF}^{+} 82\right]$ Danny Dolev, Michael J. Fischer, Rob Fowler, Nancy A. Lynch, and H. Raymond Strong. An efficient algorithm for Byzantine agreement without authentication. Information and Control, 52(3):257-274, March 1982.

[Dol82] Danny Dolev. The Byzantine generals strike again. Journal of Algorithms, 3(1):14-30, 1982.

[DS82] Danny Dolev and H. Raymond Strong. Polynomial algorithms for multiple processor agreement. In Proceedings of the 14th Annual ACM Symposium on Theory of Computing (STOC '82), pages 401-407, 1982.

[DS83] Danny Dolev and H. Raymond Strong. Authenticated algorithms for Byzantine agreement. SIAM Journal on Computing, 12(4):656-666, 1983.

$\left[\mathrm{FGH}^{+} 02\right]$ Matthias Fitzi, Daniel Gottesman, Martin Hirt, Thomas Holenstein, and Adam Smith. Detectable Byzantine agreement secure against faulty majorities. In Proceedings of the 21st ACM Symposium on Principles of Distributed Computing (PODC '02), pages 118-126, 2002.

[FHHW03] Matthias Fitzi, Martin Hirt, Thomas Holenstein, and Jürg Wullschleger. Two-threshold broadcast and detectable multi-party computation. In Eli Biham, editor, Advances in Cryptology - EUROCRYPT '03, Lecture Notes in Computer Science. Springer-Verlag, May 2003.

[FLM86] Michael J. Fischer, Nancy A. Lynch, and Michael Merritt. Easy impossibility proofs for distributed consensus problems. Distributed Computing, 1:26-39, 1986.

[FM97] Pesech Feldman and Silvio Micali. An optimal probabilistic protocol for synchronous Byzantine agreement. SIAM Journal on Computing, 26(4):873-933, August 1997.

[FM00] Matthias Fitzi and Ueli Maurer. From partial consistency to global broadcast. In Proceedings of the 32nd Annual ACM Symposium on Theory of Computing (STOC '00), pages 494-503, 2000.

[GHY87] Zvi Galil, Stuart Haber, and Moti Yung. Cryptographic computation: Secure fault-tolerant protocols and the public-key model. In Advances in Cryptology: CRYPTO '8\%, volume 293 of Lecture Notes in Computer Science, pages 135-155. Springer-Verlag, 1987.

[GL90] Shafi Goldwasser and Leonid Levin. Fair computation of general functions in presence of immoral majority. In Advances in Cryptology: CRYPTO '90, volume 537 of Lecture Notes in Computer Science, pages 11-15. Springer-Verlag, 1990.

[GL02] Shafi Goldwasser and Yehuda Lindell. Secure computation without agreement. 16th International Symposium on Distributed Computing (DISC '02). Preliminary version on http://www.research.ibm.com/people/l/lindell, 2002.

[GLR95] Li Gong, Patrick Lincoln, and John Rushby. Byzantine agreement with authentication: Observations and applications in tolerating hybrid and link faults. In Proceedings of the 5th Conference on Dependable Computing for Critical Applications (DCCA-5), pages 79-90, 1995.

[GMW87] Oded Goldreich, Silvio Micali, and Avi Wigderson. How to play any mental game. In Proceedings of the 19th Annual ACM Symposium on Theory of Computing (STOC '87), pages 218-229. ACM Press, 1987.

[Gol01] Oded Goldreich. Secure multi-party computation, working draft, version 1.3, June 2001. 
[LLR02] Yehuda Lindell, Anna Lysyanskaya, and Tal Rabin. On the composition of authenticated Byzantine agreement. In Proceedings of the 34th Annual ACM Symposium on Theory of Computing (STOC '02), pages 514-523. ACM Press, 2002.

[LSP82] Leslie Lamport, Robert Shostak, and Marshall Pease. The Byzantine generals problem. ACM Transactions on Programming Languages and Systems, 4(3):382-401, July 1982.

[PW96] Birgit Pfitzmann and Michael Waidner. Information-theoretic pseudosignatures and Byzantine agreement for $t>=n / 3$. Technical Report RZ 2882 (\#90830), IBM Research, 1996.

[RB89] Tal Rabin and Michael Ben-Or. Verifiable secret sharing and multiparty protocols with honest majority. In Proceedings of the 21st Annual ACM Symposium on Theory of Computing (STOC'89), pages 73-85, 1989.

[TC84] Russell Turpin and Brian A. Coan. Extending binary Byzantine agreement to multivalued Byzantine agreement. Information Processing Letters, 18(2):73-76, February 1984.

[WP89] Michael Waidner and Birgit Pfitzmann. Unconditional sender and recipient untraceability in spite of active attacks — some remarks. Technical Report 5/89, Universität Karlsruhe, Institut für Rechnerentwurf und Fehlertoleranz, 1989.

[Yao82] Andrew C. Yao. Protocols for secure computations. In Proceedings of the 23rd Annual IEEE Symposium on Foundations of Computer Science (FOCS '82), pages 160-164, 1982.

\section{A Reducing Broadcast to Weak Broadcast}

In this section we describe how to efficiently reduce broadcast to weak broadcast in a way that is more direct than in FM00. Given that at most $t<n / 2$ players are corrupted the resulting protocol for broadcast is as secure as the given protocol for weak broadcast.

In a first step, weak broadcast is transformed into a protocol for graded consensus (Section A.1), the "consensus variant" of graded broadcast introduced by Feldman and Micali in [FM97]; and finally, graded consensus is transformed into broadcast (Section A.2).

\section{A.1 Graded Consensus}

In graded consensus, every player has an input $x$ and receives two outputs, a value $y \in\{0,1\}$ and a grade $g \in\{0,1\}$. If all correct players start with the same value $x$ then all players output $y=x$ and $g=1$. Additionally, if any correct player ends up with grade $g=1$ then all correct players output the same value $y$, i.e., computing $g=1$ means "detecting agreement."

Definition 3 (Graded Consensus). A protocol where every player $p_{i}$ has an input $x_{i} \in\{0,1\}$ and computes two output values $y_{i}, g_{i} \in\{0,1\}$ achieves graded consensus if it satisfies the following conditions: 
- VALIDITY: If all correct players have the same input value $x$ then every correct player $p_{i}$ computes $y_{i}=x$ and $g_{i}=1$.

- Consistency: If any correct player $p_{i}$ computes $g_{i}=1$ then every correct player $p_{j}$ computes $y_{j}=y_{i}$.

The following protocol for graded consensus basically consists of two consecutive rounds wherein each player weak-broadcasts a value. Note that, in Step 4 of the protocol, the domain of weak broadcast is ternary, namely $\{0,1, \perp\}$. Following the restriction to focus on protocols with binary domains we can simply interpret such a protocol as being simulated by two parallel invocations of binary weak broadcast.

\section{Protocol 2 GradedConsensus $\left(P, x_{i}\right)$}

1. $\forall j \in\{1, \ldots, n\}: x_{i}^{j}:=$ WeakBroadcast $_{p_{j}}\left(P, x_{j}\right)$;

2. $S_{i}^{0}:=\left\{j \in\{1, \ldots, n\} \mid x_{i}^{j}=0\right\} ; S_{i}^{1}:=\left\{j \in\{1, \ldots, n\} \mid x_{i}^{j}=1\right\}$;

3. if $\left|S_{i}^{x_{i}}\right| \geq n-t$ then $z_{i}:=x_{i}$ else $z_{i}:=\perp \mathrm{fi}$;

4. $\forall j \in\{1, \ldots, n\}: z_{i}^{j}:=$ WeakBroadcast $_{p_{j}}\left(P, z_{j}\right)$;

5. $T_{i}^{0}:=\left\{j \in\{1, \ldots, n\} \mid z_{i}^{j}=0\right\} ; T_{i}^{1}:=\left\{j \in\{1, \ldots, n\} \mid z_{i}^{j}=1\right\}$;

6. if $\left|T_{i}^{0}\right|>\left|T_{i}^{1}\right|$ then $y_{i}:=0$ else $y_{i}:=1 \mathrm{fi}$;

7. if $\left|T_{i}^{y_{i}}\right| \geq n-t$ then $g_{i}:=1$ else $g_{i}=0 \mathrm{fi}$;

8. return $\left(y_{i}, g_{i}\right)$

Lemma 5 (Graded Consensus). If Protocol WeakBroadcast achieves weak broadcast then Protocol 2 achieves graded consensus secure against $t<n / 2$ corrupted players.

\section{Proof.}

VALIDITY: If all correct players hold the same value $x$ at the beginning of the protocol then, by the validity property of weak broadcast, $\left|S_{i}^{x_{i}}\right| \geq n-t>t$ for every correct player $p_{i}$ and thus $z_{i}=x_{i}=x$. Finally, $\left|T_{i}^{x}\right| \geq n-t>t$, $\left|T_{i}^{1-x}\right|<n-t$, and $y_{i}=x$ and $g_{i}=1$.

Consistency: Note that every correct player $p_{i}$ that does not compute $z_{i}=\perp$ (in Step 3) holds the same value $z_{i}=z$ : By the validity property of weak broadcast, $\left|S_{i}^{x_{i}}\right| \geq n-t$ implies that $\left|S_{j}^{1-x_{i}}\right| \leq t<n-t$.

Now, let $p_{i}$ and $p_{j}$ be two correct players and suppose that $p_{i}$ decides on $y_{i}=y \in\{0,1\}$ and $g_{i}=1$. We have to show that $y_{j}=y$.

From $g_{i}=1$ it follows that $\left|T_{i}^{y}\right| \geq n-t>t$ and thus that at least one correct player $p_{k}$ must have sent $z_{k}=y$ during Step 4 , and with the above remark, that no correct player $p_{k}$ can have sent $z_{k}=1-y$ during Step 4 .

Let $\ell$ be the number of corrupted players who distributed value $y$ during Step 4. Now, $\left|T_{i}^{y}\right| \geq n-t$ implies $\left|T_{j}^{y}\right| \geq n-t-\ell>t-\ell$ and $\left|T_{j}^{1-y}\right| \leq t-\ell$ since only the remaining $t-\ell$ corrupted players can have sent value $y$ during Step 4. Thus, $p_{j}$ computes $y_{j}:=y=y_{i}$. 


\section{A.2 Broadcast}

For simplicity, without loss of generality, assume $s=1$, i.e., that $p_{1}$ is the sender of the broadcast.

Protocol 3 Broadcast $_{p_{1}}\left(P, x_{1}\right)$
1. $p_{1}$ : Send $x_{1}$;
Receive $\left(y_{i}\right)$
2. $p_{i}$ : for $k=2$ to $t+1$ do
3. $\quad\left(y_{i}, g_{i}\right):=$ GradedConsensus $\left(P, y_{i}\right)$;
4. $\quad p_{k}$ : Send $y_{k} ; \quad \operatorname{Receive}\left(y_{i}^{k}\right)$
5. if $g_{i}=0$ then $y_{i}:=y_{i}^{k}$ fi
6. od; return $y_{i}$

Lemma 6. Suppose that Protocol GradedConsensus achieves graded consensus. If in Protocol 3, for some $k \in\{2, \ldots, t+1\}$, every correct player $p_{i}$ holds the same value $y_{i}=b$ at the beginning of Step 3 then $y_{i}=b$ holds at the end of the protocol.

Proof. Suppose that $y_{i}=b \in\{0,1\}$ for every correct player $p_{i}$ before Step 3 (for some $k$ ). Because of the validity property of graded consensus, after Step 3, (for $k$ ), every correct player $p_{i}$ holds $y_{i}=b$ and $g_{i}=1$, and thus ignores Step 5, (for $k$ ). Thus, by induction, every correct player $p_{i}$ ends the protocol with $y_{i}=b$.

Lemma 7 (Broadcast). If Protocol GradedConsensus achieves graded consensus then Protocol 3 achieves broadcast with sender $p_{1}$ (for any $t<n$ ).

Proof. We show that the validity and consistency properties of broadcast are satisfied.

VALIDITy: Suppose the sender $p_{1}$ to be correct with input $x_{s}=b$. Hence, every correct player $p_{i}$ holds value $y_{i}=b$ before Step 3 for $k=2$. And by Lemma 6 , every correct player $p_{i}$ ends the protocol with $y_{i}=b$.

CONSISTENCY: If the sender is correct then consistency is implied by the validity property. Assume now that $p_{1}$ is corrupted. Hence there is a correct player $p_{k}$ $(k \in\{2, \ldots, t+1\})$. We now argue that, for such a $k$ where $p_{k}$ is correct, every correct player $p_{i}$ holds the same value $y_{i}$ after Step 5. Then, together with Lemma 6, the consistency property follows.

First, suppose that every correct player $p_{i}$ holds $g_{i}=0$ after Step 3. Then all of them adopt $p_{k}$ 's value, $y_{i}=y_{i}^{k}$, and consistency follows from Lemma 6. Suppose now, that any correct player $p_{i}$ holds $g_{i}=1$ after Step 3 . Then, by the consistency property of graded consensus, $p_{k}$ and every other correct player $p_{j}$ hold $y_{k}=y_{i}=y_{j}$, and consistency follows from Lemma 6 .

Theorem 1. If at most $t<n / 2$ players are corrupted then efficient achievability of weak broadcast implies efficient achievability of broadcast.

Proof. Since the given construction for broadcast involves a polynomial number of invocations of weak broadcast $(2 n(t+1))$, the theorem follows directly from Lemma 7. 